\title{
New associate editor: Prof. Dr. rer.nat. Udo Jeschke
}

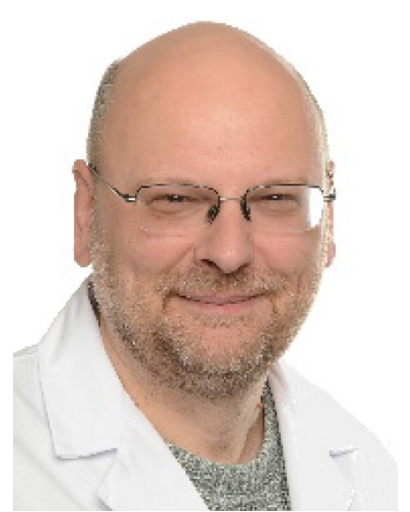

Prof. Dr. rer.nat. Udo Jeschke is currently serving as head of the Research Laboratories in the Department of Gynaecology and Obstetrics at the LMU Munich. He studied Chemistry at the University of Rostock during 1984-1989. From 1989 to 1992 he worked on his PhD thesis and could finish it in July 1992 in the field of carbohydrate chemistry.

From 1992 to 1993, he has spent a postdoctoral research period supported by the "German research Council" (DFG) at the University of Alberta, Canada. After an Internship at the Department of Medical Biochemistry of the University of Rostock, he spent a second postdoctoral research period again supported by the "German research Council" (DFG) at the University of Utrecht, The Netherlands. During that time, he could gain knowledge on the field of PregnancyProtein (PP) glycosylation analyses.
After that time, he started his research carrier at the Department of Gynaecology and Obstetrics of the University of Rostock. He received funding from the education ministry of Mecklenburg-Vorpommern. He has been serving as the head of the Research Laboratories at the Department of Obstetrics and Gynaecology from 1997 to 2002.

He has contributed to student teaching and training programs at the Department of Biology of the University of Rostock and could finish his "Habilitation" in the year 2003. He was awarded numerous awards by the "Norddeutsche Gesellschaftfür Gynäkologie und Geburtshilfe" and the "Hamburg Symposia of Tumour Marker".

His scientific focus lies on glycosylation-related signal transduction processes of pregnancy proteins, Carbohydrate-galectin interactions and molecular biology of breast, cervical, endometrial and ovarian cancer.

$\mathrm{He}$ has received numerous grants from the German Research Council (DFG) for his research and actively participated in DAAD programs for Greek-German research exchanges.

From 2002 until now, he served as the head of the research laboratories of the Department of Obstetrics and Gynaecology at the LMU Munich.

He currently serves as Board member and treasurer of the European Society of Reproductive Immunology (ESRI).

He holds an extraordinary professorship at the Medical Faculty of the LMU Munich. 